\title{
GENERALIZED BRIESKORN MANIFOLDS
}

\author{
BY RICHARD C. RANDELL
}

Communicated by Mary Ellen Rudin, July 10, 1973

Introduction. The manifolds defined by E. Brieskorn in [1] have been much studied and used in recent years. We present here the calculation of various invariants of these manifolds. Actually, we study a more general class, manifolds $K$ defined as neighborhood boundaries of isolated singularities of complete intersections of Brieskorn varieties subject to the condition that $K$ admits a natural type of $S^{1}$-action. This class contains the manifolds studied by $\mathrm{H}$. Hamm [5] as a proper subset.

The results described here are from the author's University of Wisconsin Ph.D. thesis, written with the invaluable guidance of Professor Peter Orlik. Details, proofs, and further results will appear elsewhere.

Definition. Let

$$
f_{i}\left(z_{1}, z_{2}, \ldots, z_{n+m}\right)=\sum_{j=1}^{n+m} \alpha_{i j} z_{j}^{a i j}, \quad i=1,2, \cdots, m,
$$

be a collection of complex polynomials. Let $V_{i}$ be the variety in $C^{n+m}$ associated with $f_{i}$ and let $V=\bigcap_{i=1}^{m} V_{i}$. Let $d_{i}=1$.c.m. $\left(a_{i 1}, \cdots, a_{i, n+m}\right)$, $q_{i j}=d_{i} / a_{i j}$.

We suppose

(i) $V$ is a complete intersection of the $V_{i}$.

(ii) $V$ has an isolated singularity at the origin.

(iii) $q_{i j}$ is independent of $i$ (let $q_{j}=q_{i j}$ ).

Let $K=V \cap S^{2(n+m)-1} \subset C^{n+m}$. The $C^{*}$-action on $V$ given by

$$
t \circ\left(z_{1}, \cdots, z_{n+m}\right)=\left(t^{q_{1}} z_{1}, \cdots, t^{q_{n+m}} z_{n+m}\right)
$$

restricts to an action of $S^{1}$ on $K$. Let $K^{*}$ be the orbit space, $K^{*}=K / S^{1}=$ $V-\{0\} / C^{*} . K$ is called a generalized Brieskorn manifold.

In [5] Hamm considered similar objects. He required that the exponents $a_{i j}$ be independent of $i$, a more restrictive condition than (iii) above. [4] shows that $K$ is an $(n-2)$-connected $(2 n-1)$-manifold which bounds a parallelizable manifold. Thus $K$ is essentially classified (as a smooth

AMS (MOS) subject classifications (1970). Primary 57D55, 57E25; Secondary 58G10. Key words and phrases. Brieskorn manifold, Brieskorn variety, complete intersection. 
manifold) by $H_{n-1}(K)$, a linking pairing on the torsion subgroup of $H_{n-1}(K)$, and a signature or Arf invariant (as $n$ is even or odd). (See [3], [10], and [11].)

Properties of $K^{*}$. We generalize some results of W. D. Neumann [7].

THEOREM 1. $K^{*}$ is a manifold if and only if there exist positive integers $c_{1}, \cdots, c_{m} ; t_{1}, \cdots, t_{n+m} ; s_{1}, \cdots, s_{n+m}$ such that

(i) $\left(t_{j}, t_{k}\right)=1, j \neq k$;

(ii) $\left(s_{j}, t_{j}\right)=1$, all $j$;

(iii) g.c.d.( $\left(s_{j_{1}}, \cdots, s_{j_{m+1}}\right)=1$ for all $(m+1)$-element subsets $\left\{j_{1}, \cdots\right.$, $\left.j_{m+1}\right\}$ of $\{1, \cdots, n+m\}$;

(iv) $a_{i j}=\left(c_{i} t_{j} / s_{j}\right) 1$. c.m. $\left(s_{1}, \cdots, s_{n+m}\right)$, for all $i$ and $j$.

It turns out that $t_{j}=$ g.c.d. $\left(q_{1}, \cdots, \hat{q}_{j}, \cdots, q_{n+m}\right)$.

By a slight generalization. of the construction of Brieskorn and Van de Ven [2], $K^{*}$ has a (possibly singular) complex structure.

THEOREM 2. $K^{*}$ is a manifold if and only if this complex structure is nonsingular.

THEOREM 3. $K^{*}$ (but not $K$ ) is independent of the $t_{j}$.

Hence we assume that $t_{j}=1$ when we work with $K^{*}$.

Next we turn to index invariants of $K^{*}$. First we prove a general result. Let $X$ be a compact complex manifold and let

$$
G=\boldsymbol{Z}_{n_{1}}\left\langle\beta_{1}\right\rangle \oplus \cdots \oplus \boldsymbol{Z}_{n_{k}}\left\langle\beta_{k}\right\rangle
$$

act effectively on $X$ so that

(i) the fixed point set $Y_{i}$ of $\left(1, \cdots, \beta_{i}, \cdots, 1\right) \in G$ has complex codimension 1 ,

(ii) the various $Y_{i}$ 's intersect transversely, and

(iii) the fixed point set of $\left(1, \cdots, \beta_{i_{1}}^{k_{1}}, \cdots, \beta_{i_{s}}^{k_{s}}, \cdots, 1\right), \beta_{i_{j}}^{k_{j}} \neq 1$, $j=1, \cdots, s$, is $Y_{i_{1}} \cap \cdots \cap Y_{i_{s}}$.

Let sign, $\chi$, and $e$ denote signature, arithmetic genus, and euler characteristic respectively.

THEOREM 4.

$$
\begin{aligned}
\operatorname{sign}(X / G) & =\operatorname{sign}\left(\prod_{i=1}^{k} \frac{\left(1+Y_{i}\right)^{n_{i}}+\left(1-Y_{i}\right)^{n_{i}}}{\left(1+Y_{i}^{n_{i}}-\left(1-Y_{i}\right)^{n_{i}}\right.} Y_{i}\right) ; \\
\chi(X / G) & =\chi\left(\prod_{i=1}^{k} \frac{1}{1-\left(1-Y_{i}\right)^{n_{i}}}\right) ; \\
e(X / G) & =e\left(\prod_{i=1}^{k}\left(\frac{1}{n_{i}}+\frac{\left(n_{i}-1\right)}{n_{i}} Y_{i}\right)\right) .
\end{aligned}
$$


This theorem is to be interpreted as in Hirzebruch [6]. The proof uses some of the techniques of that paper.

We apply Theorem 4 and similar results to compute the index invariants of $K^{*}$. We find a manifold $X$ and a finite group $G$ so that $X / G \cong K^{*}$. For this $X$ and $G$ it is easy to compute the index invariants of monomials in the $Y_{i}$, and thus we can compute the invariants of $K^{*}$.

Here is an example. Let $D=d_{1} d_{2} \cdots d_{m}, Q=q_{1} q_{2} \cdots q_{n+m}$. Let $r_{s}$ be the coefficient of $x^{s}$ in $\prod_{i=1}^{m}\left(1-d_{i} x+d_{i}^{2} x^{2}-\cdots\right)$, and let $J=\{1, \cdots, n+m\}$, $J_{k}=\left\{j_{1}, \cdots, j_{k}\right\} \subset J$.

THEOREM 5.

$$
\begin{aligned}
e\left(K^{*}\right)= & \frac{D}{Q}\left\{\left[\sum_{k=0}^{n-1}\left(\sum_{s=0}^{n-1-k}\left(r_{s}\left(\begin{array}{c}
n+m-k \\
n-1-s-k
\end{array}\right) \sum_{J k} \prod_{J} \prod_{i=1}^{k}\left(q_{j_{i}}-1\right)\right)\right)\right]\right. \\
+ & {\left[\sum_{J k} \subset J, k>m\right.} \\
& \left.\left.\left.\left(\text { g.c.d. }\left(q_{j_{1}}, \cdots, q_{j_{k}}\right)-1\right) \sum_{s=0}^{k-1-m} r_{s}(k-m-1-s)\right)\right]\right\} .
\end{aligned}
$$

(For this formula the $a_{i j}$ must be normalized so that the $t_{j}$ 's are 1.)

The homology of $K$ and $K^{*}$. The $S^{1}$-action has no fixed points on $K$, so the orbit map $\pi: K \rightarrow K^{*}$ has a rational Gysin sequence. From this we obtain

THEOREM 6. Let $\kappa=\operatorname{rank}\left(\tilde{H}_{n-1}(K ; Z)\right)$.

(i) $H_{q}\left(K^{*} ; Q\right) \cong\left\{\begin{array}{ll}Q, & q \text { even }, 0 \leqq q \leqq 2 n-2 \\ 0, & \text { otherwise }\end{array}\right\} \oplus\left\{\begin{array}{ll}Q^{\kappa}, & q=n-1 \\ 0, & \text { otherwise }\end{array}\right\}$;

$$
\kappa=(-1)^{n-1}\left(e\left(K^{*}\right)-n\right) .
$$

Thus Theorems 5 and 6 describe the rational homology of $K$ and $K^{*}$.

We conclude with an algorithm for computing the torsion of $H_{*}(K)$ and $H_{*}\left(K^{*}\right)$. It is a generalization for Orlik's conjecture for the homology torsion of Brieskorn manifolds [8].

$A=\left(a_{i j}\right)$ is an $m \times(n+m)$-matrix. Let $A\left(j_{1}, \cdots, j_{s}\right)$ be the $m \times s$ matrix consisting of columns $j_{1}, \cdots, j_{s}$ of $A$. Let $K\left(j_{1}, \cdots, j_{s}\right)=\left\{z \in K \mid z_{j}=0\right.$ for $\left.j \notin\left\{j_{1}, \cdots, j_{s}\right\}\right\}$. Let $\kappa(K)=\kappa(A)=\operatorname{rank}\left(\tilde{H}_{n-1}(K)\right)$, and $\kappa\left(A\left(j_{1}, \cdots, j_{s}\right)\right)=$ $\operatorname{rank}\left(\widetilde{H}_{s-m-1}\left(K\left(j_{1}, \cdots, j_{s}\right)\right)\right)$. Let $\kappa$ of the empty matrix be 1 . Put

$$
\begin{aligned}
& \varepsilon_{n+m-s}=1, \text { if } n+m-s \text { is odd, } \\
& =0, \text { if } n+m-s \text { is even; } \\
& \varepsilon_{n+m-s, q}=1 \text {, if } 0<s+1-m \leqq q \text { and } q+m-s \text { is odd, } \\
& =0 \text {, otherwise. }
\end{aligned}
$$


Let $c(\varnothing)=$ g.c.d. $\left(a_{11}, \cdots, a_{1, n+m}\right)$ and for $J_{s}=\left\{j_{1}, \cdots, j_{s}\right\}$ with $j_{1}<\cdots<j_{s}$ define

$$
c\left(j_{1}, \cdots, j_{s}\right)=\frac{\text { g.c.d. }\left(a_{11}, \cdots, \hat{a}_{1, j_{1}}, \cdots, \hat{a}_{1, j_{s}}, \cdots, a_{1, n+m}\right)}{\Pi_{I} \subsetneq J_{s} c\left(i_{1}, \cdots, i_{t}\right)}
$$

where $I=\left\{i_{1}, \cdots, i_{t}\right\}$ and $i_{1}<\cdots<i_{t}$. Except for $c(\varnothing)$ the $c\left(j_{1}, \cdots, j_{s}\right)$ are independent of the row of $A$ used to compute them. (Here we used the first row.) We redefine $c(\varnothing)$ by

Let

$$
c(\varnothing)=\prod_{i=1}^{m} \text { g.c.d. }\left(a_{i 1}, \cdots, a_{i, n+m}\right) \cdot \prod_{t<m} c\left(i_{1}, \cdots, i_{t}\right) .
$$

$$
\begin{gathered}
k\left(j_{1}, \cdots, j_{s}\right)=\varepsilon_{n+m-s} \kappa\left(j_{1}, \cdots, j_{s}\right), \\
k_{q}\left(j_{1}, \cdots, j_{s}\right)=\varepsilon_{n+m-s, q} \kappa\left(j_{1}, \cdots, j_{s}\right), \\
d_{j}=\prod_{k\left(j_{1}, \cdots, j_{s}\right) \geqq j} c\left(j_{1}, \cdots, j_{s}\right), \quad d_{j, q}=\prod_{k_{q}\left(j_{1}, \cdots, j_{s}\right) \geqq j} c\left(j_{1}, \cdots, j_{s}\right), \\
r=\max \left\{k\left(j_{1}, \ldots, j_{s}\right)\right\}, \quad \text { and } r_{q}=\max \left\{k_{q}\left(j_{1}, \cdots, j_{s}\right)\right\} .
\end{gathered}
$$

Let $\operatorname{Tor}(A)=\boldsymbol{Z}_{d_{1}} \oplus \cdots \oplus \boldsymbol{Z}_{d_{r}}$ and $\operatorname{Tor}_{q}(A)=\boldsymbol{Z}_{d_{1}, q} \oplus \cdots \oplus \boldsymbol{Z}_{d_{r_{q}, q}}$ and for any finitely generated abelian group $G$, let $\operatorname{Tor}(G)$ denote the torsion subgroup.

THEOREM 7. (i) $\operatorname{Tor}\left(H_{n-1}(K)\right) \cong \operatorname{Tor}(A)$;

$$
\operatorname{Tor}\left(H_{q}\left(K^{*}\right)\right) \cong \begin{cases}\operatorname{Tor}_{q}(A), & q \leqq n-2 \\ 0, & \text { otherwise }\end{cases}
$$

For $m=1$ (i.e. K Brieskorn), (i) was conjectured by Orlik.

Sketch OF PROOF. We use induction on $n$. For $n=1$ the result is trivial. For $n=2$ it follows from [9], so we assume the result for $\operatorname{dim}(K)<$ $2 n-1$ with $n>2$. Let $Z(p)$ denote the integers localized at some prime ideal $(p)$. First suppose that all $t_{j}=1$. Since $t_{j}=$ g.c.d. $\left(q_{1}, \cdots, \hat{q}_{j}, \cdots, q_{n+m}\right)$, $L_{p}=\left\{z \in K \mid\left(p,\left|S_{z}^{1}\right|\right)>1\right\}$ is a submanifold of codimension at least 4 . Let $L \supset L_{p}$ have codimension equal to 4 . $\left(L=K \cap\left\{z_{i_{1}}=z_{i_{2}}=0\right\}\right)$. Let $N$ be a closed $S^{1}$-invariant tubular neighborhood of $L$, and let $M=(K-N)^{-}$, with $\pi: M \rightarrow M^{*}$ the orbit map. $\pi$ has a Gysin sequence over $Z(p)$ and $M^{*}$ satisfies Poincaré duality over $Z(p)$. We use these facts to show that there are isomorphisms of $p$-torsion $H_{n-3}(L) \cong H_{n}(\partial M) \cong H_{n}(M) \cong H_{n-1}\left(M^{*}\right) \cong$ $H_{n-1}(M) \cong H_{n-1}(K)$. By comparing the results of the algorithm for $H_{n-3}(L)$ (known by inductive hypothesis) and $H_{n-1}(K)$ we find that the $p$-torsion of $H_{n-1}(K)$ is given by the algorithm. Finally we relax the assumption that $t_{j}=1$. If $p$ divides $t_{j}$, we note that $K$ is a $t_{j}$-fold branched cover of $K^{\prime}$, where $K^{\prime}$ has $t_{j}=1$. By comparing the difference of the torsion of $K$ 
with the (known) torsion of $K^{\prime}$ we complete the induction step for (i). For (ii) we use similar ideas.

\section{REFERENCES}

1. E. Brieskorn, Beispiele zur Differentialtopologie von Singularitäten, Invent. Math. 2 (1966), 1-14. MR 34 \#6788.

2. E. Brieskorn and A. Van de Ven, Some complex structures on products of homotopy spheres, Topology 7 (1968), 389-393. MR 38 \#1682.

3. A. H. Durfee, Diffeomorphism classification of isolated hypersurface singularities, Ph.D. Thesis, Cornell University, Ithaca, N.Y., 1971.

4. H. Hamm, Lokale topologische Eigenschaften komplexer Räume, Math. Ann. 191 (1971), 235-252. MR 44 \#3357.

5. - Exotische Sphären als Umgebungsränder in speziellen komplexen Räumen, Math. Ann. (1972), 44-56.

6. F. Hirzebruch, The signature of ramified coverings, Global Analysis (Papers in Honor of K. Kodaira), Univ. Tokyo Press, Tokyo, 1969, pp. 253-265. MR 41 \#2707.

7. W. D. Neumann, $S^{1}$-actions and the $\alpha$-invariant of their involutions, Bonner Mathematische Schriften 44, 1970.

8. P. Orlik, On the homology of weighted homogeneous manifolds, Proc. Second Conf. Transformation Groups. I, Lecture Notes in Math, no. 298, Springer-Verlag, Berlin and New York, 1972, pp. 260-269.

9. $\mathrm{P}$. Orlik and $\mathrm{P}$. Wagreich, Isolated singularities of algebraic surfaces with $C^{*}$ action, Ann. of Math. 93 (1971), 205-228.

10. C. T. C. Wall, Classification problems in differential topology. VI: Classification of (s-1)-connected (2s+1)-manifolds, Topology 6 (1967), 273-296. MR 35 \#7343.

11. D. L. Wilkens, Closed ( $s-1)$-connected $(2 s+1)$-manifolds, $s=3$, 7, Bull. London Math. Soc. 4 (1972), 27-31.

Department of Mathematics, University of Wisconsin, Madison, Wisconsin 53706

Current address: Department of Mathematics, University of Michigan, Ann Arbor, Michigan 48104 